\title{
Parental and social factors in relation to child psychopathology, behavior, and cognitive function
}

\author{
Han Zhang (1)', Zu Xuan Lee', Tonya White ${ }^{2}$ and Anqi Qiu (iD
}

\begin{abstract}
Parental and social factors have long-term impact on the neurodevelopment of offspring, but tend to highly covary with each other. Thus, it is difficult to parse out which parental and social factor contributes most to neurodevelopmental outcomes. This study aimed to assess clusters of parental and social factors associated with child psychopathology, behavioral problems, and cognition. This study employed the data of 11,875 children (9 to 11 years) from the Adolescent Brain Cognitive Development (ABCD) study. Principal component analysis (PCA) was performed on 39 environmental measures and 30 child behavior and cognitive measures separately to identify clusters of parental and social factors and clusters of child psychopathology, behaviour, and cognition. Regression analysis was used to examine independent effects of each cluster of parental and social factors on child psychopathology, behavioral problems, and cognition. Greater Parent Psychopathology cluster was associated with greater Child Psychopathology cluster. Moreover, greater Socioeconomic Status cluster was associated with greater child General Cognition and Executive Function but less Behavioral Inhibition clusters. Greater Proximal Social Environment and Interaction cluster were associated with less child Impulsive Behavior and Behavioral Inhibition, but greater Behavioral Activation cluster. The environmental clusters related to birth outcomes, maternal tobacco, and drug use were not significantly related to child psychopathology, behavior, and cognition. Our findings suggest that socioeconomic status, parental psychopathology, and social environment and interactions are the strongest risks for behavioral problems and cognitive performance in a general child population. Intervention programs should target modifiable factors within these domains.
\end{abstract}

\section{Introduction}

Parental, socioeconomic, and social factors, such as parent psychopathology, pregnancy complications, household income, parental education, and family environment, can have long-term impact on the neurodevelopment of offspring ${ }^{1-4}$. However, most of existing studies typically assess parental, socioeconomic, and social factors, and their influences on child psychopathology, behavior, and cognition, separately. These environmental factors not only play an important role in neurodevelopment, but also tend to covary highly with each other, which makes it difficult to parse out which parental and

\footnotetext{
Correspondence: Anqi Qiu (bieqa@nus.edu.sg)

${ }^{1}$ Department of Biomedical Engineering, National University of Singapore, Singapore, Singapore

${ }^{2}$ Department of Child and Adolescent Psychiatry, Erasmus University Medical Center - Sophia, Children's Hospital, Rotterdam, The Netherlands
}

social factor contributes most to neurodevelopmental outcomes, or whether the risk is additive ${ }^{5,6}$.

The Adolescent Brain Cognitive Development (ABCD) study (version 2.0) acquired comprehensive information on prenatal and postnatal parental, socioeconomic, and social environment as well as child outcomes in 11,875 children aged at 9 to 11 years $^{7}$. It provided a unique opportunity to assess each aspect of parental, socioeconomic, and psychosocial factors in relation with child psychopathology, behavioral problems, and cognition when considering the interplay of different aspects of parental and social factors. For this, we employed principal component analysis to identify clusters within a wide spectrum of parental, socioeconomic, and social environmental factors and clusters within a wide spectrum of child psychopathology, behavioral problems, and cognition. Such an approach provides a comprehensive map for 
understanding the contribution of individual aspects of parental, socioeconomic and social factors to child psychopathology, behavioral problems, and cognition, which potentially provides the guidance of future intervention on improving child neurodevelopment in a general population.

\section{Methods}

\section{Participants}

Participant data were obtained from the open baseline from the ongoing Adolescent Brain Cognitive Development (ABCD) study (release 2.0; https://abcdstudy.org/). Youth $(n=11,875) 9-11$ years of age were recruited for this study and formed a similar proportion of males and females living in the United States. The sample selection criteria were targeted to reflect the sociodemographic proportion of the U.S. population as described in the ABCD study design ${ }^{7}$. All participants were administered assessments to obtain data on the respective youth's brain morphology, cognitive function, substance use, demographics, and environment ${ }^{8}$. Written informed consent was obtained from all parents, and all children provided assent to a research protocol approved by the institutional review board at each data collection site (https:// abcdstudy.org/study-sites/( $)^{9}$.

Of the 11,875 participants, we excluded 23 subjects with missing values of demographics, 3219 subjects with one or more missing values of the parental and social environmental measures, 914 subjects with one or more missing values of the questionnaires/tasks of the child psychopathology, behavior, and cognitive measures, and 283 subjects with missing values in either of these two data. Therefore, our study employed 11,875 participants and 8002 participants (67.4\% of full sample) for statistical analysis, separately. Supplementary Table S1 in the Supplementary Material lists the subject id whose data were not included in this study.

\section{Parental and social environmental measures}

This study included 39 parental, socioeconomic, and social environmental measures, including10 measures of parent psychopathology, 6 maternal substance use measures, 5 developmental adversity measures, 7 social demographics, 5 proximal environmental measures, and 6 social interaction measures ${ }^{8,10}$.

\section{Parent psychopathology}

Parent psychopathology symptoms were assessed using the Adult Self Report (ASR) and Family History Assessment Module Screener (FHAM-S) questionnaires. The ASRprovides 8 empirically-based syndrome scales (anxious/depressed, withdrawn, somatic complaints, thought problems, attention problems, aggressive behaviour, rule-breaking behavior, and intrusive) ${ }^{11}$. FHAM-S reports the presence/absence of symptoms associated with alcohol and drug use, depression, and mania in all 1st and 2nd degree "blood relatives" of the youth ${ }^{12}$. The presence of alcohol and drug use problems of the child's relatives was defined as the family psychopathology risk of substance use disorders. Similarly, the accumulated presence of depression and mania was scored as the family psychopathology risk of mental disorders.

\section{Maternal substance use}

The parent-reported Developmental History Questionnaire was used to assess maternal consumption of tobacco, alcohol, and marijuana before and after the mothers knew that they were pregnancy ${ }^{13-15}$.

\section{Developmental adversity}

The developmental History Questionnaire ${ }^{8}$ was used to assess prematurity, birth weight, pregnancy and birth complications and the Modified Ohio State University Traumatic Brain Injury Screen-Short Version ${ }^{16}$ was employed to assess the parent-report overall brain injury/ concussion during the child's development.

\section{Social demographics}

The parent-report demographics battery from the PhenX toolkit measured social demographics of the parental highest education, household annual income, and marriage status ${ }^{17}$. Economic insecurity ${ }^{18}$, the grand total Uniform Crime Reports, Area Deprivation Index by the scaled weighted sum, and the estimated lead risk in census tract of primary residential address ${ }^{19,20}$ were also employed to provide additional information about socioeconomic influences.

\section{Proximal environment}

The "Safety from Crime" items from the PhenX Toolkit was used to assess neighborhood safety and crime reports $^{21,22}$. Additionally, children reported their school risk and protective factors via a 12-item Inventory for School Risk and Protective Factors of the PhenX toolkit ${ }^{23}$. Three measures was selected to assess a child's connectedness to his/her school, including school teacher and classroom environment, personal involvement in school, and alienation from academic goals.

\section{Social interaction}

The child-reported parental monitoring and acceptance, as well as the child- and parent-reported prosocial tendency and family conflicts were included to measure social interactions. Parent monitoring was accessed by a 5-item summary score of the Parental Monitoring Scale ${ }^{24}$. Parent acceptance was evaluated by the Acceptance Scale, a subscale of the Child Report of Behavior Inventory $(\mathrm{CRPBI})^{25}$. Prosocial behavior (e.g., being nice, helping, 


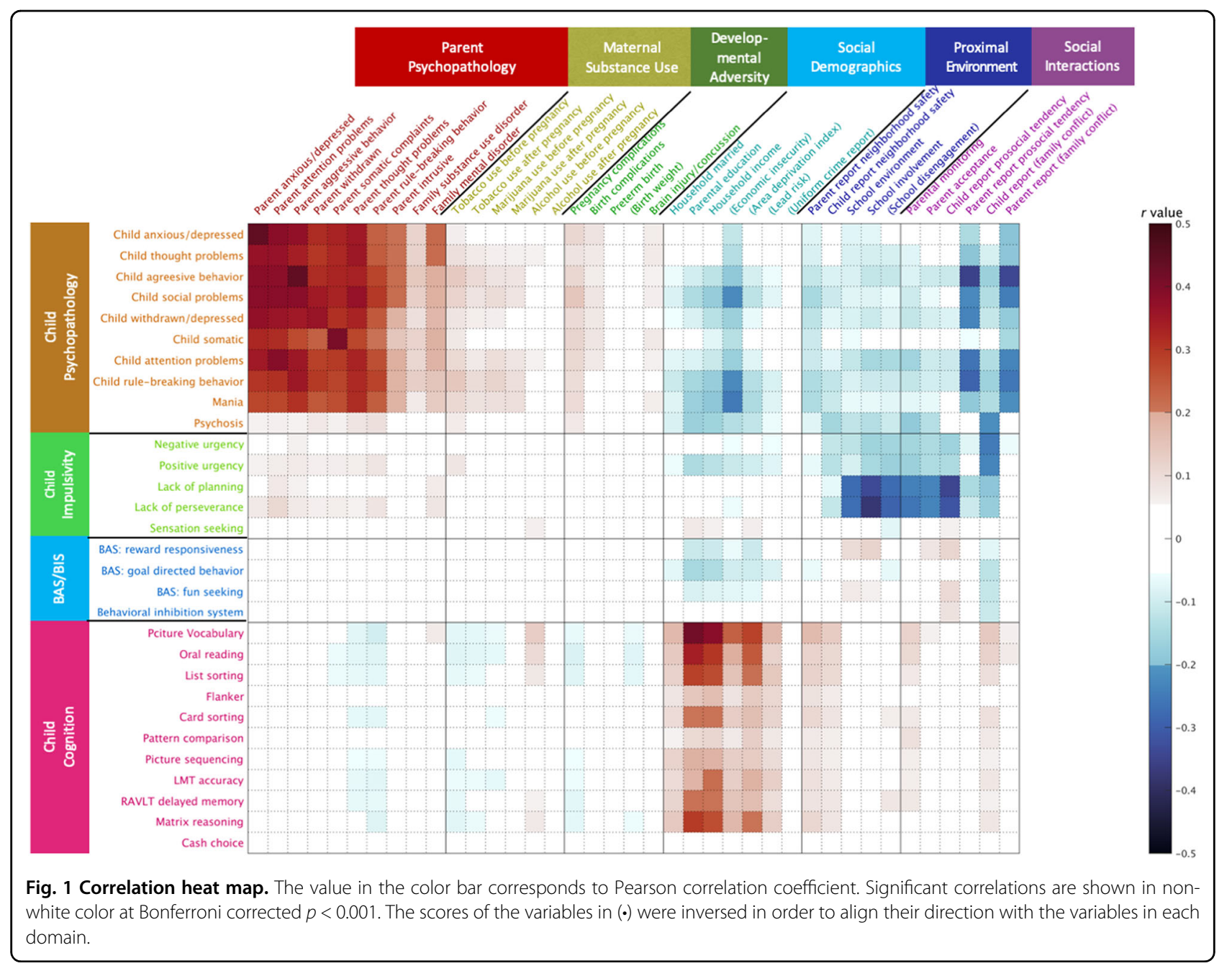

caring) was assessed using the Prosocial Behavior Scale, a subscale from the "Strengths and Difficulties Questionnaire" (SDQ) ${ }^{26}$. Both parents and youth reported on the youth's prosocial behavior (e.g., being considerate of other people's feelings, often offering to help others). In order to assess the family conflicts, the $A B C D$ protocol utilized a 9-item Family Conflict subscale of the Moos Family Environment Scale (FES) for the baseline protocol $^{27}$.

For the parental and environmental measures related to psychopathology, maternal substance use, and developmental adversity, higher scores represents more severe psychiatric symptoms, worse substance use, and developmental adversity. For the measures of social demographics, proximal environment, and social interactions, higher scores represent better socioeconomic status, proximal environment, and social interactions. For the ease of interpretation, a few scores were inverted to align the direction in their same category as mentioned above. Figure 1 marks these inverted measures in parenthesis.

\section{Child psychopathology, behavior, and cognition}

This study employed 30 child psychopathology, behavior, and cognitive measures, including 10 child psychopathology measures, 9 behavior measures, and 11 cognitive measures ${ }^{8,28}$. To provide converging evidence about the youth's behavior, we also utilized the available data $(n=2440)$ with the teach-reported total behavior problems which were evaluated by the Brief Problem Monitor-Teacher Form ${ }^{11}$.

\section{Child psychopathology}

Child Psychopathology was assessed based on the parent report of Child Behavior Checklist $(\mathrm{CBCL})^{11}$, the tenitem Mania Scale derived from the Parent General Behavior Inventory for Children and Adolescents ${ }^{29}$, and the Prodromal Questionnaire Brief Version ${ }^{30}$. This study included 8 empirically-based syndrome scales from CBCL (aggressive behavior, anxious/depressed, attention problems, rule-breaking behavior, somatic complaints, social problems, thought problems, and withdrawn/depressed scales), a risk score of bipolar variability in mood and 
behaviour, and a severity score of psychosis risk symptoms.

\section{Child behavior}

The 20-item Children-Short Form (UPPS-P) was used to assess five facets of impulsivity ${ }^{31}$, including negative and positive urgency, lack of planning, lack of perseverance, and sensation seeking. The 24-item Behavioral Inhibition/Activation Scales (BIS/BAS) were also utilized: BIS (e.g., worry, fearfulness), BAS drive (intensity of goal directed behavior), BAS reward responsiveness (excitement over reinforcing outcomes), and BAS fun seeking (enjoyment for its own sake, spontaneity) ${ }^{32}$.

\section{Child cognition}

The neurocognitive battery comprised of 11 tasks $^{28}$ and was administered using an iPad with one-on-one monitoring by a research assistant. Among the 11 cognitive tasks, there were 7 from the NIH Toolbox (http://www. nihtoolbox. org), including flanker (inhibitory control), dimensional change card sort (cognitive flexibility), list sorting working memory (working memory), picture sequence memory (episodic memory), pattern comparison processing speed (processing speed), picture vocabulary (vocabulary comprehension), and oral reading recognition tasks (reading decoding). ABCD also administered Matrix Reasoning Task from the Wechsler Intelligence Test for Children-V (fluid Reasoning) ${ }^{33}$, Little Man Task (LMT, visual-spatial processing), Rey Auditory Verbal Learning Test (RAVLT, auditory learning, memory, and recognition), and Cash Choice Task (a singleitem delayed gratification measure with dichotomous scoring). Notably, we employed the response accuracy of LMT, the delayed recall accuracy of RAVLT, and the total scaled score of Matrix Reasoning.

For measures related to child psychopathology and behavior, a higher score represented worse psychopathology and behavioral problems. For measures of child cognition, a higher score represented better cognitive ability.

\section{Statistical analysis}

Each score of 39 environmental measures and 30 child characteristics was first standardized with zero mean and unit variance using rank-based inverse Gaussian transformation $^{33,34}$. Pearson's correlation coefficients were used to explore the associations of individual parental and social environment variables with individual child measures. Bonferroni correction was used to determine the significance of multiple correlations (the number of tests: 1170) at $p<0.001$.

For multivariate analysis, principal component analyses (PCA) was first performed within all environmental measures and within the child characteristics, respectively $^{33}$. Varimax rotation was applied to factor loadings of the PCs with eigenvalues greater than 1 . The component scores were further computed based on the varimax rotated loadings beyond 0.35 . This procedure ensured statistical independence of the PCs within the environmental measures and within the child characteristics.

Mixed effect models were used to examine associations of all environmental PCs with each child characteristic PCs. Age, sex and ethnicity were covariates. The information of twins, non-twin siblings, and 21 different research sites was entered as random effects. Bonferroni correction was used to determine the significance of statistical tests $(n=48)$ at $p<0.001$.

\section{Results}

This study included 8022 out of 11,875 children (mean [SD] age, 9.9 [0.6] years; $47.8 \%$ girls; $57.0 \%$ white ethnicity) with the complete environmental and child characteristic data. Table 1 lists the 39 environmental measures and 30 child characteristics of subjects with the complete data $(n=8002)$ and all 11,875 subjects. The sample with the complete data $(n=8022)$ did not differ from the whole sample $(n=11,875)$ in most of measures. However, some environmental measures (i.e., household married percentage, parental education, lead risk, and parent report neighborhood safety) and cognitive measures (i.e., picture vocabulary, oral reading, list sorting, card sorting, picture sequencing, RAVLT delayed memory and matrix reasoning) were better in the sample with the complete data than the whole sample (see $p$-values in Table 1). The severity of child psychopathology (i.e., child aggressive behavior, child attention problems, and child rule-breaking behavior from CBCL, and the mania score). was slightly lower in the sample with the complete data than in the whole sample data (see $p$-values in Table 1).

Figure 1 illustrates significant correlations between 39 parental and social environment measures and 30 child outcomes (Bonferroni corrected $p<0.001$ ). This suggested strong correlations between parental and child psychopathology, between socioeconomic status and cognition, between social interactions and child psychopathology, and between proximal social environment and interactions and child impulsive behaviors.

Figure 2 shows 8 PCs for environmental factors (48.7\% variance explained) and 6 PCs for child characteristics (51.6\% variance explained). The $8 \mathrm{PC}$ environmental factors included (1) Parent Psychopathology (14.3\% variance explained), Socioeconomic Status (7.5\% variance explained), (3) Proximal social environment and interaction (7.3\% variance explained), Birth Outcomes (5.1\% variance explained), (5) Maternal Tobacco Use (4.7\% variance explained), (6) Neighbourhood Safety (3.5\% variance explained), (7) Family Psychopathology (3.3\% variance explained), and (8) Maternal Marijuana Use (3.0\% variance explained). The 6 PC child characteristic components included (1) Child Psychopathology (17.9\% variance 
Table 1 Demographics, parental and social environmental measures and child outcomes for complete data and full sample data.

\begin{tabular}{|c|c|c|c|}
\hline & $\begin{array}{l}\text { Complete data } \\
\text { mean (SD) }\end{array}$ & $\begin{array}{l}\text { Full sample data* } \\
\text { mean (SD) }\end{array}$ & $p$ \\
\hline Age & $9.9(0.6)$ & $9.9(0.6)$ & 0.954 \\
\hline Gender (\%) & & & 0.971 \\
\hline Male & 52.2 & 52.1 & \\
\hline Female & 47.8 & 47.9 & \\
\hline Race/ethinicity (\%) & & & 0.000 \\
\hline White & 57.0 & 52.1 & \\
\hline Black & 12.2 & 15.0 & \\
\hline Hispanic & 19.0 & 20.3 & \\
\hline Asian & 2.0 & 2.1 & \\
\hline Other & 9.8 & 10.5 & \\
\hline Parent anxious/depressed & $5.0(4.9)$ & $5.0(4.9)$ & 0.268 \\
\hline Parent attention problems & $4.6(4.2)$ & $4.6(4.3)$ & 0.283 \\
\hline Parent aggressive behavior & $3.3(3.5)$ & $3.4(3.6)$ & 0.086 \\
\hline Parent withdrawn & $1.5(2.0)$ & $1.6(2.1)$ & 0.007 \\
\hline Parent somatic complaints & $2.8(3.0)$ & $2.9(3.2)$ & 0.041 \\
\hline Parent thought problems & $1.4(1.7)$ & $1.4(1.9)$ & 0.010 \\
\hline Parent rule-breaking behavior & $1.1(1.8)$ & $1.2(1.9)$ & 0.006 \\
\hline Parent intrusive & $1.0(1.4)$ & $1.0(1.4)$ & 0.759 \\
\hline Family substance use disorder & $0.8(0.8)$ & $0.8(0.8)$ & 0.480 \\
\hline Family mental disorder & $2.5(2.1)$ & $2.5(2.1)$ & 0.267 \\
\hline Tobacco use before pregnancy & $0.91(3.3)$ & $1.0(3.6)$ & 0.011 \\
\hline Tobacco use after pregnancy & $0.3(1.8)$ & $0.3(2.0)$ & 0.036 \\
\hline $\begin{array}{l}\text { Marijuana use before } \\
\text { pregnancy }\end{array}$ & $0.1(0.7)$ & $0.1(0.7)$ & 0.603 \\
\hline Marijuana use after pregnancy & $0.02(0.24)$ & $0.02(0.26)$ & 0.505 \\
\hline Alcohol use before pregnancy & $0.9(2.6)$ & $0.9(2.7)$ & 0.631 \\
\hline Alcohol use after pregnancy & $0.04(0.66)$ & $0.05(1)$ & 0.175 \\
\hline Pregnancy complications & $0.7(1)$ & $0.7(1.1)$ & 0.298 \\
\hline Birth complications & $0.4(0.8)$ & $0.4(0.8)$ & 0.309 \\
\hline Preterm birth & $0.9(2.2)$ & $0.9(2.2)$ & 0.960 \\
\hline Birth weight (lbs) & $6.6(1.5)$ & $6.6(1.5)$ & 0.078 \\
\hline Brain injury/concussion & $1.1(0.3)$ & $1.1(0.3)$ & 0.937 \\
\hline Household married (\%) & 82.7 & 80.7 & 0.000 \\
\hline Parental education (\%) & & & 0.000 \\
\hline$<$ HS diploma & 3.5 & 5.0 & \\
\hline HS diploma/GED & 7.8 & 9.5 & \\
\hline Some college & 24.8 & 26.0 & \\
\hline Bachelor & 27.1 & 25.4 & \\
\hline Post graduate degree & 36.8 & 34.1 & \\
\hline Household income (\%) & & & 0.004 \\
\hline$[<50 \mathrm{~K}]$ & 27.5 & 29.7 & \\
\hline$[\geq 50 \mathrm{~K} \&<100 \mathrm{~K}]$ & 28.6 & 28.3 & \\
\hline$[\geq 100 \mathrm{~K}]$ & 43.9 & 42.1 & \\
\hline Economic insecurity & $0.4(1.0)$ & $0.4(1.1)$ & 0.002 \\
\hline Area deprivation index & $92.0(25.0)$ & $93.0(25.0)$ & 0.102 \\
\hline Lead risk & $4.9(3.1)$ & $5.1(3.1)$ & 0.000 \\
\hline Uniform crime report & $49,000(81,000)$ & $52,000(85,000)$ & 0.008 \\
\hline $\begin{array}{l}\text { Parent report } \\
\text { neighborhood safety }\end{array}$ & $3.9(0.9)$ & $3.9(1.0)$ & 0.000 \\
\hline $\begin{array}{l}\text { Child report } \\
\text { neighborhood safety }\end{array}$ & $4.1(1.1)$ & $4(1.1)$ & 0.005 \\
\hline School environment & $20.0(2.7)$ & $20.0(2.8)$ & 0.461 \\
\hline School involvement & $13.0(2.3)$ & $13.0(2.4)$ & 0.412 \\
\hline School disengagement & $3.7(1.4)$ & $3.7(1.5)$ & 0.177 \\
\hline Parental monitoring & $4.4(0.5)$ & $4.4(0.5)$ & 0.038 \\
\hline
\end{tabular}

Table 1 continued

\begin{tabular}{|c|c|c|c|}
\hline & $\begin{array}{l}\text { Complete data } \\
\text { mean (SD) }\end{array}$ & $\begin{array}{l}\text { Full sample data* } \\
\text { mean (SD) }\end{array}$ & $p$ \\
\hline Parent acceptance & $2.8(0.3)$ & $2.8(0.3)$ & 0.073 \\
\hline $\begin{array}{l}\text { Child report prosocial } \\
\text { tendency }\end{array}$ & $1.7(0.4)$ & $1.7(0.4)$ & 0.458 \\
\hline $\begin{array}{l}\text { Parent report prosocial } \\
\text { tendency }\end{array}$ & $1.8(0.39)$ & $1.8(0.4)$ & 0.036 \\
\hline Child report (family conflict) & $2.0(1.9)$ & $2.0(2.0)$ & 0.115 \\
\hline Parent report (family conflict) & $2.5(1.9)$ & $2.5(2.0)$ & 0.110 \\
\hline Child anxious/depressed & $2.5(3.0)$ & $2.5(3.1)$ & 0.546 \\
\hline Child thought problems & $1.6(2.1)$ & $1.6(2.2)$ & 0.073 \\
\hline Child agreesive behavior & $3.0(4.1)$ & $3.3(4.4)$ & 0.000 \\
\hline Child social problems & $1.5(2.2)$ & $1.6(2.3)$ & 0.001 \\
\hline Child withdrawn/depressed & $1.0(1.6)$ & $1.0(1.7)$ & 0.025 \\
\hline Child somatic & $1.5(1.9)$ & $1.5(2.0)$ & 0.299 \\
\hline Child attention problems & $2.8(3.4)$ & $3(3.5)$ & 0.000 \\
\hline Child rule-breaking behavior & $1.1(1.7)$ & $1.2(1.9)$ & 0.000 \\
\hline Mania & $1.2(2.5)$ & $1.3(2.8)$ & 0.000 \\
\hline Psychosis & $5.9(10.0)$ & $6.3(11.0)$ & 0.003 \\
\hline Negative urgency & $8.4(2.6)$ & $8.5(2.6)$ & 0.157 \\
\hline Positive urgency & $7.9(2.9)$ & $8(3)$ & 0.016 \\
\hline Lack of planning & $7.7(2.4)$ & $7.7(2.4)$ & 0.906 \\
\hline Lack of perseverance & $7.0(2.2)$ & $7.0(2.3)$ & 0.139 \\
\hline Sensation seeking & $9.8(2.7)$ & $9.8(2.7)$ & 0.427 \\
\hline BAS: reward responsiveness & $8.8(2.4)$ & $8.8(2.4)$ & 0.484 \\
\hline BAS: goal directed behavior & $4.0(3.0)$ & $4.1(3.1)$ & 0.001 \\
\hline BAS: fun seeking & $5.7(2.6)$ & $5.7(2.6)$ & 0.386 \\
\hline Behavioral inhibition system & $5.5(2.8)$ & $5.5(2.8)$ & 0.246 \\
\hline Pciture Vocabulary & $85.0(8.0)$ & $84.0(8.1)$ & 0.000 \\
\hline Oral reading & $91.0(6.7)$ & $91.0(6.9)$ & 0.000 \\
\hline List sorting & $98.0(12.0)$ & $97.0(12.0)$ & 0.000 \\
\hline Flanker & $94.0(8.9)$ & $94.0(9.1)$ & 0.002 \\
\hline Card sorting & $93.0(9.2)$ & $93.0(9.5)$ & 0.000 \\
\hline Pattern comparison & $88.0(14.0)$ & $88.0(15.0)$ & 0.119 \\
\hline Picture sequencing & $100.0(12.0)$ & $100(12.0)$ & 0.000 \\
\hline LMT accuracy & $0.6(0.2)$ & $0.6(0.2)$ & 0.003 \\
\hline RAVLT delayed memory & $9.3(3.1)$ & $9.2(3.2)$ & 0.000 \\
\hline Matrix reasoning & $10.0(2.9)$ & $9.9(3.0)$ & 0.000 \\
\hline Cash choice & $1.6(0.5)$ & $1.6(0.5)$ & 0.253 \\
\hline
\end{tabular}

Group differences are tested using two-sample t-test with equal variance assumption for continuous variables and $x^{2}$ tests for discrete variables. *Due to missing values, the sum of percentages may not equal to $100 \%$.

explained), General Cognition (11.6\% variance explained), (3) Behavioral Activation (8.3\% variance explained), Impulsive Behavioral Problems (5.6\% variance explained), (5) Executive Function (4.3\% variance explained), and (6) Behavioral Inhibition (3.9\% variance explained).

After controlling for age, gender, ethnicity, and the other environmental PC factors, greater Parent Psychopathology (standardized $\beta=0.56,[0.54,0.58], p<0.001$ ) was associated with greater Child Psychopathology (Fig. 3a). Using available reports on the Teacher Report Form on child behaviors $(n=2440)$, the associations between Parent Psychopathology and teacher-reported child behavioral problems remained significant (standardized $\beta=0.06,[0.02,0.11], p=0.002$ ). Moreover, greater 


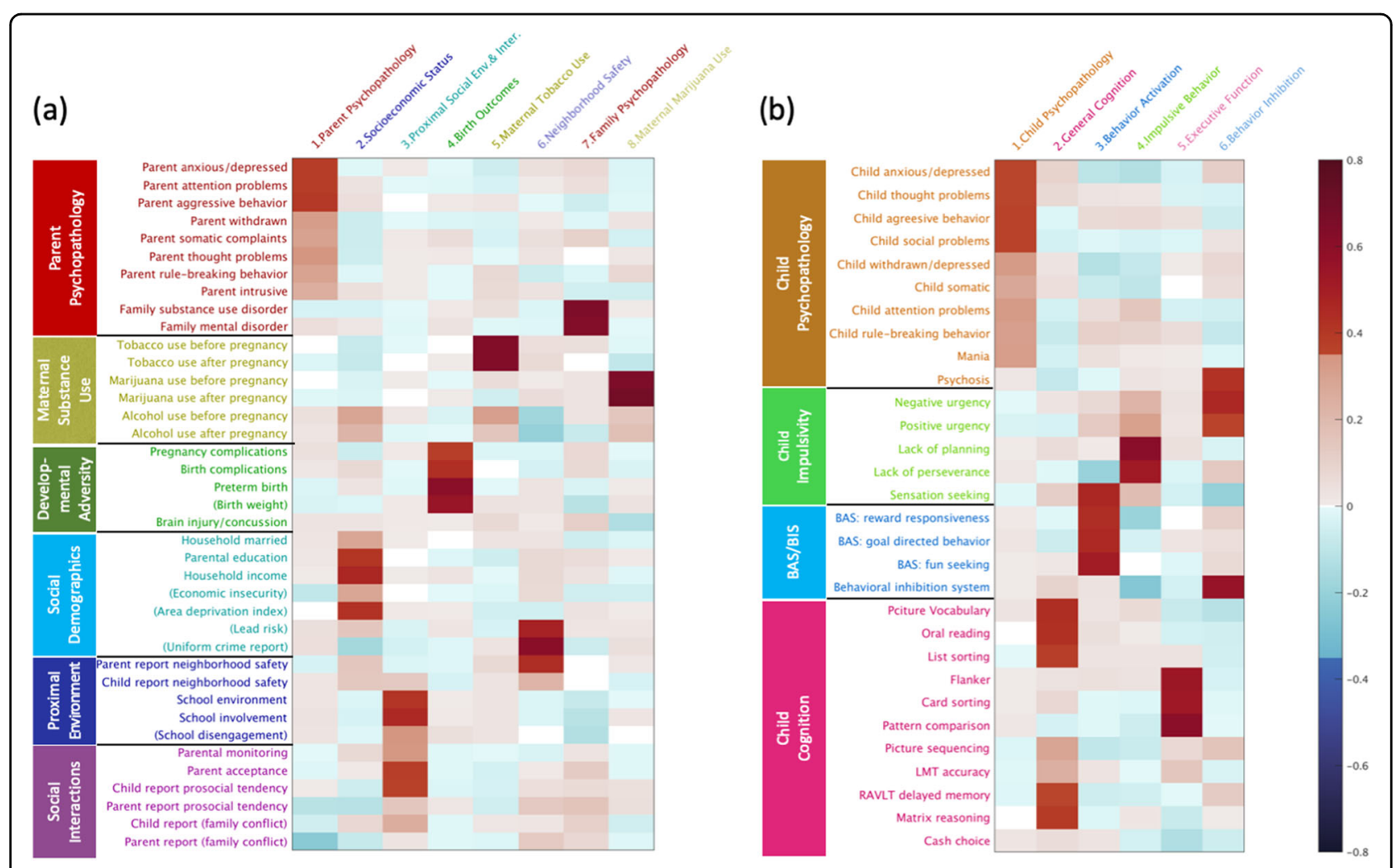

Fig. 2 Varimax rotated loadings of retained principal components. a Parental, socioeconomic, and social environmental factors have 8 components retained. b Child characteristics have 6 components retained. BAS, behavioral activation system; BIS, behavioral inhibition system.

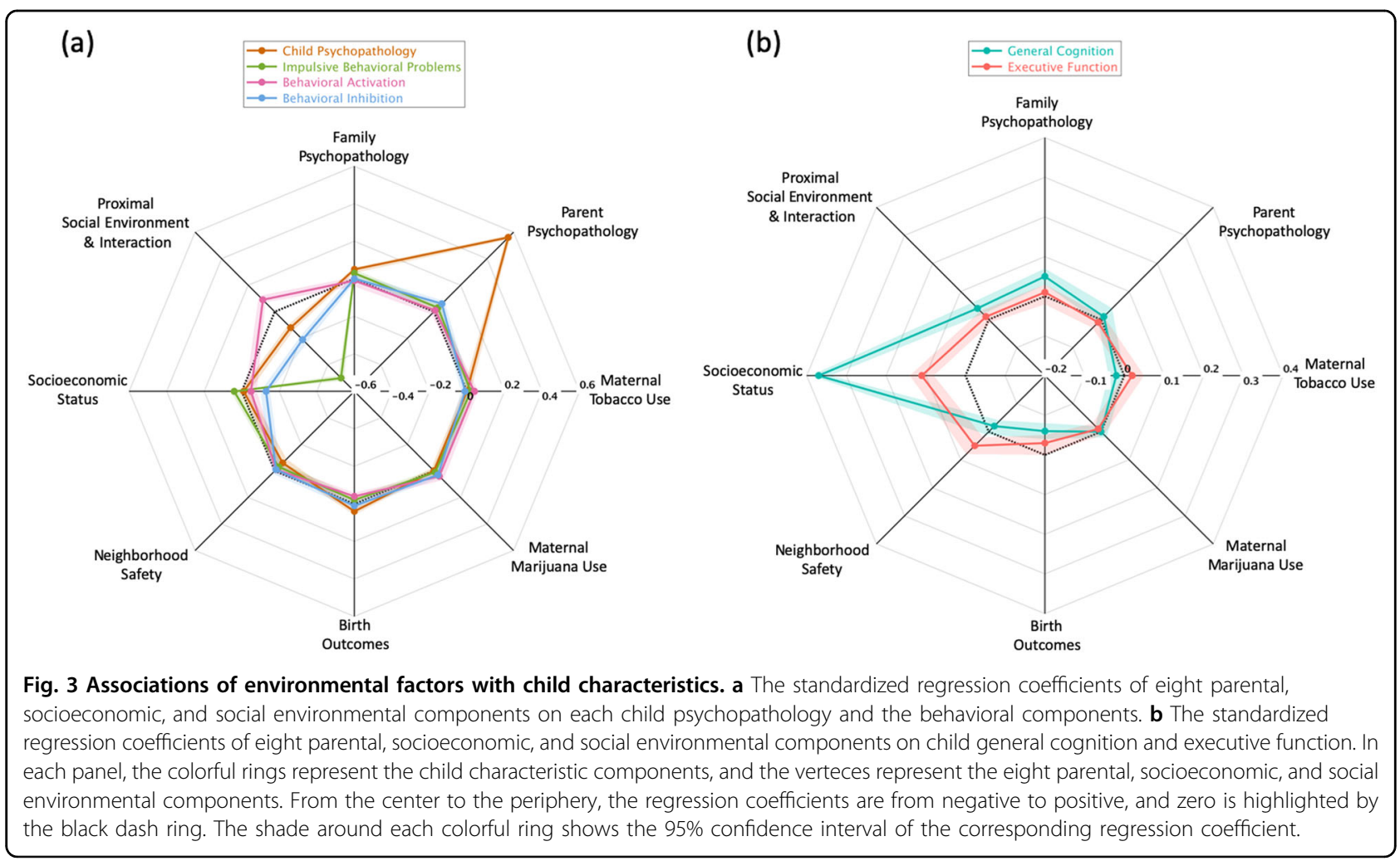


Socioeconomic Status was associated with greater child General Cognition (standardized $\beta=0.37,[0.34,0.39], p<$ 0.001 ) and Executive Function (standardized $\beta=0.11$, [0.08, 0.14], $p<0.001$, Fig. 3b) but with less Behavioral Inhibition (standardized $\beta=-0.13,[-0.16,-0.10], p<$ 0.001; Fig. 3a). Greater Proximal Social Environment and Interaction were associated with less child Impulsive Behavioral Problems (standardized $\beta=-0.50,[-0.52$, $-0.48], p<0.001$ ) and Behavioral Inhibition (standardized $\beta=-0.21,[-0.24,-0.19], p<0.001)$, but greater Behavioral Activation (standardized $\beta=0.09,[0.07,0.12], p<$ 0.001; Fig. 3a). The environmental PCs related to birth outcomes, maternal alcohol, tobacco, and drug use were not significantly related to child psychopathology, behavior, and cognition (Tables 2, 3).

Our repeated analyses using the full study sample $(n=$ 11,875) and mean imputation for missingness showed the similar findings as stated above (in Supplementary Figs. S1 and S2 of the Supplementary Material).

\section{Discussion}

This study showed the distinctive influences of the parental, socioeconomic, and social environmental factors on child psychopathology, behavioral problems, and cognition. As expected, strong relationships were found between Parent Psychopathology and Child Psychopathology, between Socioeconomic Status and child Cognition, and between Proximal Social Environment and Interaction and child Impulsive behaviors. What was unexpected, however, was our lack of identifying relationships between birth outcomes, maternal tobacco and drug use with child psychopathology, behavioral problems, and cognition.

Consistent with previous findings ${ }^{35,36}$, we found strong association between the psychopathology in parents and their children. Child psychopathology was assessed by parents and thus there is a tendency that parents with greater psychopathology will also rate their child as having greater psychopathology. When we utilized teacher reported behavioral problems of the child and parent selfreport, the association remained significant, albeit less strong. Our findings provide further support for a potential genetic contribution for the transgenerational transmission of psychopathology from parents to behavioral characteristics of children.

This study also identified the associations of Socioeconomic Status with child General Cognition and Executive Function. This is congruent with previous findings, suggesting that lower Socioeconomic Status strongly predicts lower IQ and executive functions ${ }^{3,37}$. Most of previous studies employ household income and/ or parental education or both as the representation of Socioeconomic Status ${ }^{20}$. In contrast, we quantified Socioeconomic Status using a broad construct that incorporated 
Table 3 Associations of all environmental PCs with each child cognitive PCs.

\begin{tabular}{|c|c|c|c|c|}
\hline \multirow[t]{2}{*}{ Variables } & \multicolumn{2}{|l|}{ General cognition } & \multicolumn{2}{|l|}{ Executive function } \\
\hline & Standardized $\beta(95 \% \mathrm{Cl})$ & $p$ & Standardized $\beta(95 \% \mathrm{Cl})$ & $p$ \\
\hline Parent psychopathology & $0.01(-0.01$ to 0.04$)$ & 0.164 & $-0.01(-0.03$ to 0.01$)$ & 0.434 \\
\hline Socioeconomic status & 0.37 (0.34 to 0.39$)$ & $0.000^{*}$ & 0.11 (0.08 to 0.14$)$ & $0.000^{*}$ \\
\hline Proximal social env. \& inter. & $0.04(0.02$ to 0.06$)$ & $0.000^{*}$ & $0.01(-0.01$ to 0.03$)$ & 0.355 \\
\hline Birth outcomes & $-0.06(-0.08$ to -0.04$)$ & $0.000^{*}$ & $-0.03(-0.05$ to 0$)$ & 0.025 \\
\hline Maternal tobacco use & $-0.02(-0.04$ to 0$)$ & 0.021 & 0.02 (0 to 0.05$)$ & 0.051 \\
\hline Neighborhood safety & $-0.02(-0.04$ to 0.01$)$ & 0.199 & 0.05 (0.02 to 0.08$)$ & 0.001 \\
\hline Family psychopathology & 0.05 (0.03 to 0.07$)$ & $0.000^{*}$ & $0.01(-0.02$ to 0.03$)$ & 0.546 \\
\hline Maternal marijuana use & $0.00(-0.02$ to 0.02$)$ & 0.977 & $-0.01(-0.03$ to 0.01$)$ & 0.349 \\
\hline
\end{tabular}

*The significant results with Bonferroni corrected $p<0.01$.

variation not only from household income and parental education, but also from a regional deprivation index. From this aspect, our study provided evidence supporting the idea of a reduction of poverty and increasing education at the level of both family and neighbourhood may help improve child cognitive development.

Unlike previous studies ${ }^{38,39}$, our findings did not support strong associations of birth outcomes, maternal tobacco, and drug use with child psychopathology, behavior, and cognition in this general child population. Nevertheless, when analyzing the association between maternal marijuana use and psychosis, we showed the similar result $(p=0.014$ in Table 2$)$ as that presented in ${ }^{15}$. The lack of such associations among the PC scores is partly because our findings were obtained after controlling for Parental Psychopathology, Socioeconomic Status, and etc, suggesting that Parental Psychopathology and Socioeconomic Status had a greater effect on child neurodevelopmental outcomes. Most of existing studies generally focus only on a case-control or imbalanced designs and do not assess the comprehensive profile of parental, socioeconomic, and social factors and hence may not quantify true effects of maternal tobacco and drug use as well as birth outcomes on child neurodevelopment in a general population ${ }^{40}$.

One of the strengths of our study is that we employed a large population-based sample of children who are all participating in the ABCD baseline wave of data collection. Thus, we were able to incorporate a comprehensive assessment of parental, socioeconomic, and social environmental factors as well as child characteristics. Nevertheless, the reliance on cross-sectional data precludes any determination of causality. Moreover, the ABCD study sampled from the United States, which may limit the generalizability of our findings. Further research is necessary to explore across other ethnicities and cultures to enhance the potential generalization of our findings.

Our findings suggest that parental psychopathology, socioeconomic status, and social environment and interactions are the strongest risks for behavioral problems and cognitive performance in a general child population. These children should be targeted for intervention programs, with the possibility for including both primary and secondary prevention.

\section{Acknowledgements}

This research is supported by the Singapore Ministry of Education (Academic research fund tier 1; NUHSRO/2017/052/T1-SRP-Partnership/01), and NUS Institute of Data Science, Singapore. Data used in the preparation of this article were obtained from the Adolescent Brain Cognitive Development (ABCD) Study (https://abcdstudy.org), held in the NIMH Data Archive (NDA). This is a multisite, longitudinal study designed to recruit more than 10,000 children age 9-10 and follow them over 10 years into early adulthood. The ABCD Study is supported by the National Institutes of Health and additional federal partners under awards U01DA041022, U01DA041025, U01DA041028, U01DA041048, U01DA041089, U01DA041093, U01DA041106, U01DA041117, U01DA041120, U01DA041134, U01DA041148, U01DA041156, U01DA041174, U24DA041123, and U24DA041147. A full list of supporters is available at https://abcdstudy.org/ federal-partners/. A listing of participating sites and a complete listing of the study investigators can be found at https://abcdstudy.org/study-sites/. ABCD consortium investigators designed and implemented the study and/or provided data but did not necessarily participate in analysis or writing of this report. This manuscript reflects the views of the authors and may not reflect the opinions or views of the $\mathrm{NIH}$ or $\mathrm{ABCD}$ consortium investigators. The $\mathrm{ABCD}$ data repository grows and changes over time. The $A B C D$ data used in this report came from https://doi.org/10.15154/1517592.

Conflict of interest

The authors declare that they have no conflict of interest.

\section{Publisher's note}

Springer Nature remains neutral with regard to jurisdictional claims in published maps and institutional affiliations.

Supplementary Information accompanies this paper at (https://doi.org/ 10.1038/s41398-020-0761-6). 
Received: 22 October 2019 Accepted: 7 February 2020

Published online: 26 February 2020

\section{References}

1. Wang, Q. et al. Sex-dependent associations among maternal depressive dymptoms, child reward network, and behaviors in early childhood. Cereb. Cortex https://doi.org/10.1093/cercor/bhz135 (2019).

2. Clark, C. A., Woodward, L. J., Horwood, L. J. \& Moor, S. Development of emotional and behavioral regulation in children born extremely preterm and very preterm: biological and social influences. Child Dev. 79, 1444-1462 (2008).

3. Noble, K. G. et al. Family income, parental education and brain structure in children and adolescents. Nat. Neurosci. 18, 773-778 (2015).

4. Almas, A. N. et al. The impact of caregiving disruptions of previously institutionalized children on multiple outcomes in late childhood. Child Dev. 91, 96-109 (2020).

5. Potijk, M. R., Kerstjens, J. M., Bos, A. F., Reijneveld, S. A. \& de Winter, A. F. Developmental delay in moderately preterm-born children with low socioeconomic status: risks multiply. J. Pediatr. 163, 1289-1295 (2013).

6. Harnish, J. D., Dodge, K. A. \& Valente, E. Mother-child interaction quality as a partial mediator of the roles of maternal depressive symptomatology and socioeconomic status in the development of child behavior problems. Conduct problems prevention research group. Child Dev. 66, 739-753 (1995).

7. Garavan, $H$. et al. Recruiting the $A B C D$ sample: design considerations and procedures. Dev. Cogn. Neurosci. 32, 16-22 (2018).

8. Barch, D. M. et al. Demographic, physical and mental health assessments in the adolescent brain and cognitive development study: Rationale and description. Dev. Cogn. Neurosci. 32, 55-66 (2018).

9. Clark, D. B. et al. Biomedical ethics and clinical oversight in multisite observational neuroimaging studies with children and adolescents: The ABCD experience. Dev. Cogn. Neurosci. 32, 143-154 (2018).

10. Zucker, R. A. et al. Assessment of culture and environment in the Adolescent Brain and Cognitive Development Study: Rationale, description of measures, and early data. Dev. Cogn. Neurosci. 32, 107-120 (2018).

11. Achenbach, T. Achenbach System of Empirically Based Assessment (ASEBA): Development, Findings, Theory, and Applications. Encyclopedia of Autism Spectrum Disorders.

12. Rice, J. P. et al. Comparison of direct interview and family history diagnoses of alcohol dependence. Alcohol. Clin. Exp. Res. 19, 1018-1023 (1995).

13. Kessler, R. C. et al. Design and field procedures in the US National Comorbidity Survey Replication Adolescent Supplement (NCS-A). Int. J. Methods Psychiatr. Res. 18, 69-83 (2009).

14. Kessler, R. C. et al. National comorbidity survey replication adolescent supplement (NCS-A): II. Overview and design. J. Am. Acad. Child Adolesc. Psychiatry 48, 380-385 (2009).

15. Fine, J. D. et al. Association of prenatal cannabis exposure with psychosis proneness among children in the Adolescent Brain Cognitive Development (ABCD) Study. JAMA Psychiatry 4-6 https://doi.org/10.1001/ jamapsychiatry.2019.0076 (2019).

16. Bogner, J. A. et al. Test-retest reliability of traumatic brain injury outcome measures. J. Head. Trauma Rehabil. 32, E1-E16 (2017).

17. Hamilton, C. M. et al. The PhenX Toolkit: get the most from your measures. Am. J. Epidemiol. 174, 253-260 (2011).

18. Diemer, M. A., Mistry, R. S., Wadsworth, M. E., López, I. \& Reimers, F. Best practices in conceptualizing and measuring social class in psychological research Anal. Soc. Issues Public Policy 13, 77-113 (2013).

19. Kind, A. J. H. et al. Neighborhood socioeconomic disadvantage and 30-day rehospitalization. Ann. Intern. Med. 161, 765 (2014).
20. Farah, M. J. The neuroscience of socioeconomic status: correlates, causes, and consequences. Neuron 96, 56-71 (2017).

21. Echeverria, S. E. Reliability of self-reported neighborhood characteristics. J. Urban Heal. Bull. N. Y. Acad. Med. 81, 682-701 (2004).

22. Mujahid, M. S., Diez Roux, A. V., Morenoff, J. D. \& Raghunathan, T. Assessing the measurement properties of neighborhood scales: from psychometrics to ecometrics. Am. J. Epidemiol. 165, 858-867 (2007).

23. Stover, P. J., Harlan, W. R., Hammond, J. A., Hendershot, T. \& Hamilton, C. M. PhenX: A toolkit for interdisciplinary genetics research. Curr. Opin. Lipidol. 21, 136-140 (2010).

24. Chilcoat, H. D. \& Anthony, J. C. Impact of parent monitoring on initiation of drug use through late childhood. J. Am. Acad. Child Adolesc. Psychiatry 35, 91-100 (1996).

25. Schaefer, E. S. A configurational analysis of children's reports of parent behavior. J. Consult. Psychol. 29, 552-557 (1965).

26. Goodman, R., Meltzer, H. \& Bailey, V. The strengths and difficulties questionnaire: a pilot study on the validity of the self-report version. Int. Rev. Psychiatry 15, 173-177 (2003).

27. Moos, R. H. \& Moos, B. S. Family Environment Scale manual. (Consulting Psychologists Press, Palo Alto, CA, 1986).

28. Luciana, M. et al. Adolescent neurocognitive development and impacts of substance use: Overview of the adolescent brain cognitive development (ABCD) baseline neurocognition battery. Dev. Cogn. Neurosci. 32, 67-79 (2018).

29. Youngstrom, E. A., Frazier, T. W., Demeter, C., Calabrese, J. R. \& Findling, R. L. Developing a 10-Item mania scale from the parent general behavior inventory for children and adolescents. J. Clin. Psychiatry 69, 831-839 (2008).

30. Loewy, R. L., Therman, S., Manninen, M., Huttunen, M. O. \& Cannon, T. D. Prodromal psychosis screening in adolescent psychiatry clinics. Early Interv. Psychiatry 6, 69-75 (2012).

31. Zapolski, T. C. B., Stairs, A. M., Settles, R. F., Combs, J. L. \& Smith, G. T. The measurement of dispositions to rash action in children. Assessment 17, 116-125 (2010).

32. Carver, C. S. \& White, T. L. Behavioral inhibition, behavioral activation, and affective responses to impending reward and punishment: The BIS/BAS Scales. J. Pers. Soc. Psychol. 67, 319-333 (1994).

33. Thompson, W. K. et al. The structure of cognition in 9 and 10 year-old children and associations with problem behaviors: Findings from the ABCD study's baseline neurocognitive battery. Dev. Cogn. Neurosci. 36, 100606 (2019).

34. Miller, K. L. et al. Multimodal population brain imaging in the UK Biobank prospective epidemiological study. Nat. Neurosci. 19, 1523-1536 (2016).

35. Befera, Mschulte \& Barkley, R. A. Hyperactive and normal girls and boys: mother-child interaction, parent psychiatric status and child psychopathology. J. Child Psychol. Psychiatry 26, 439-452 (1985).

36. Gunlicks, M. L. \& Weissman, M. M. Change in child psychopathology with improvement in parental depression: a systematic review. J. Am. Acad. Child Adolesc. Psychiatry 47, 379-389 (2008).

37. Turkheimer, E., Haley, A., Waldron, M., D'Onofrio, B. \& Gottesman, I. I. Socioeconomic status modifies heritability of IQ in young children. Psychol. Sci. 14, 623-628 (2003).

38. Bhutta, A. T., Cleves, M. A., Casey, P. H., Cradock, M. M. \& Anand, K. J. S. Cognitive and behavioral outcomes of school-aged children who were born preterm. JAMA 288, 728 (2002).

39. Huizink, A. C. \& Mulder, E. J. H. Maternal smoking, drinking or cannabis use during pregnancy and neurobehavioral and cognitive functioning in human offspring. Neurosci. Biobehav. Rev. 30, 24-41 (2006).

40. Huizink, A. C. Prenatal cannabis exposure and infant outcomes: overview of studies. Prog. Neuro-Psychopharmacol. Biol. Psychiatry 52, 45-52 (2014). 